\title{
Grupos estratégicos: su influencia en el desempeño de la industria bancaria venezolana y su relación con la cobertura geográfica
}

\section{Strategic groups: their influence on the performance of the Venezuelan banking industry and its relationship with the geographic coverage}

\author{
Sandra Lizbeth Flores Márquez' y José Ovidio Flores Gutiérrez² \\ ' Depto. Ingeniería de Organización, Administración de Empresa y Estadística, E.T.S Ingenieros Industriales, Universidad Politécnica de Madrid. \\ C/José Gutiérrez Abascal, 2.28006 Madrid, España. \\ 2 UNELLEZ-Guanare. Antiguo Convento de San Francisco, carrera 3 entre carrera 16 y 17, Mesa de Cavaca, Guanare, Estado Portuguesa. \\ Venezuela. \\ sandralizbethflores@gmail.com, joseovidioflores@gmail.com
}

Fecha de recepción: 12-5-2011

Fecha de aceptación: 16-12-2011

Resumen: El objetivo de esta investigación fue determinar los grupos estratégicos de la industria bancaria venezolana y su influencia sobre el desempeño en el sector, así como su relación con la cobertura geográfica, durante el primer semestre del año 2008. Los resultados más relevantes fueron los siguientes: I) Los grupos estratégicos combinan patrones de conductas. 2) Las diferentes estrategias adoptadas por los bancos no se expresaron en una diferenciación en los niveles de resultados económicos y financieros. 3) Los grupos bancarios llevan a cabo una estrategia de cobertura geográfica relacionada con la estrategia financiera.

Palabras clave: grupos estratégicos, banca, desempeño, cobertura geográfica.

Abstract: The objective of this research was to determine the strategic groups Venezuelan banking industry are their influence on performance and its relationship to geographic coverage, throughout the first half of 2008. This period was selected for two reasons: 1) This semester was characterized, in Latin America as being a period of economic stability before the global financial crisis and 2) The General Law of Banks and Other Financial Institutions requires banks to publish their financial statements each semester. The sample consisted of 52 financial institutions. The data required to carry out this research was obtained from the balance sheets and income statements published by ASOBANCA. Before application of cluster analysis to the 8 variables that integrate the scope of the strategy, we conducted a principal components analysis to determine the relationship between these variables and to detect outliers, while distinguishing the strategies that characterized the group following the common procedure used by Amel and Rhoades proposed (1988), and was reinforced by the realization of parametric statistical and non-parametric tests. The use of these tests was to identify novel strategies followed by strategic groups in banking and allow, in contrast to commonly used methods to verify the existence of statistically different groups as well as homogeneous groups who develop a common strategy. To determine the performance differences between strategc groups regarding financial and economic performance variables, we applied the contrasts of ANOVA and Kruskal-Wallis. We used SPSS for tabulation of data and to apply the analysis and the tests described above while evaluating the geographical coverage of banking and demonstrate the link between it and the strategic groups using the software Geographic Information System ArcGIS. Finally, we used the Spearman test to analyze the coverage of strategic groups in the less poor municipalities and those considered most attractive for investment. The results were as follows: I) Banks employ different patterns of strategic behavior. 2) The combined strategic group behavior patterns. This paper identified five strategic groups: conservative banking, commercial banking, retail banking, corporate and public small corporate banking. 3) The different strategies adopted by banks were not expressed in a differentiation in levels of economic and financial results. 4) The banking groups a strategy of geographic coverage relating to their financial strategy. Thus the conservative bank concentrates its offices in the municipalities that are less risky and more profitable. The commercial banks locate $81.82 \%$ of its offices in those cities where the bank generally gives the largest volume of loans to microentrepreneurs. Retail banking has the largest network of offices. Corporate public bank networks includes offices moderate some of the less poor municipalities, but also situated in the poorest municipalities, which shows greater social responsibility, consistent with the mission of public enterprises. Small corporate banking, located in Chacao, close to most of the bank headquarters that make up the other strategic groups, make it easy for their strategy of placing their funds in the portfolio, which includes lending to other financial institutions.

Key words: strategic groups, banking, performance, geographic coverage. 


\section{Introducción}

La presente investigación tiene como objetivo determinar los grupos estratégicos de la industria bancaria venezolana y su influencia sobre el desempeño en el sector, así como su relación con la cobertura geográfica. Este objetivo principal se confía en conseguir a través de la consecución de los cuatro objetivos específicos siguientes: Determinar las relaciones entre las variables de alcance de la estrategia, identificar y caracterizar los distintos grupos estratégicos en la industria bancaria venezolana, analizar las diferencias de desempeño entre los grupos estratégicos obtenidos y describir la relación entre la cobertura geográfica y los grupos estratégicos.

Para identificar los grupos estratégicos se utilizaron las dimensiones estratégicas de alcance y recursos comprometidos propuestas por Cool y Schendel ( 1987), que han sido ampliamente estudiadas por investigadores en diferentes industrias, destacando en el sector bancario las investigaciones de Mas (1994, 1996), Surroca (2003) y Almanza y Daccarett (2005).

Posteriormente a la conformación de los grupos estratégicos, se aborda la relación entre los grupos estratégicos y la cobertura geográfica, considerando esta última dimensión como la red de oficinas que posee cada banco, en los ámbitos de los distintos municipios del país, cuyo despliegue se evidenció empleando los sistemas de información geográficos (SIG). La correspondencia entre los grupos estratégicos y la cobertura geográfica de los mismos, desde la perspectiva de los SIG, ha sido poco estudiada, por lo cual, esta investigación contribuirá al conocimiento de la relación de las estrategias de alcance y recursos comprometidos con la elección de los mercados geográficos.

Por otra parte, es necesario señalar que el interés por estudiar la banca surge por las razones siguientes:

- Del éxito en el funcionamiento del sector financiero depende en gran medida el crecimiento económico y el bienestar social en cada país.

- Tanto en Venezuela como en otras partes del mundo, se ha producido un historial de crisis financieras que han llevado a la quiebra a varios bancos, generando perjuicios a muchos ahorristas y clientes en general.

- La banca venezolana es objeto de continuas amenazas de nacionalizaciones, lo que genera rumores, y a su vez provocan incertidumbre y temor, especialmente, a los ahorristas, por la posibilidad de perder su dinero.

- Existe una crisis financiera mundial, que ha influido en las oscilaciones de los precios del petróleo, fuente casi exclusiva de los ingresos del Estado venezolano, quien es el principal cliente y deudor de la banca venezolana, particularmente por la elevada colocación de títulos de la Deuda Pública Nacional (Blaser, 2004).

En el mismo orden de ideas, los resultados de esta investigación permitirán a los gerentes de entidades financieras, conocer cuáles son las estrategias que permiten obtener una mayor rentabilidad en su organización sobre las de sus competidores así como las implicaciones estratégicas de la cobertura geográfica de las redes bancarias. Con estos conocimientos pueden tomar decisiones acertadas que permitan hacer más rentable su empresa, y de la misma manera, determinar hacia qué zonas es mejor expandirse a través de sucursales.

También a los interesados en adquirir acciones de las entidades bancarias que cotizan en la Bolsa de Valores de Caracas, les permite comparar las estrategias que desarrolla cada banco, y saber cuales tienen mayor influencia en los resultados financieros.

Por su parte, al Estado venezolano, que es propietario de algunas de las entidades bancarias en el país, le interesa conocer, no solo las estrategias que hacen más rentables a organizaciones de este tipo, sino además en que municipios la presencia de las entidades financieras es elevada y en cuales no existen entidades bancarias, es decir, cuales zonas se caracterizan por la exclusión geográfica financiera como forma de exclusión social (Papillón et al., 2004, p. 19; Gwinner et al., 2006), para tomar los correctivos necesarios y promover el desarrollo económico y social de esas regiones, con el fin de obtener la rentabilidad social que debe caracterizar a las instituciones del Estado.

\section{Revisión de la literatura}

\section{I. Descripción de la estructura del sistema bancario venezolano a partir de 1994}

La crisis financiera venezolana ocurrida en enero del año 1994 fue la expresión de una crisis social, política, de las políticas públicas y hasta de valores (Borgucci, 200l) y, trajo, entre otras consecuencias, la 
apertura al capital extranjero. Esto generó mejoras a nivel técnico y operativo, además del fortalecimiento en el patrimonio de las entidades financieras (Bracho et al., 2002, p. 303).

Pero también la crisis financiera dio origen a un nuevo marco regulatorio, que buscaba mejorar la calidad de la supervisión y de la regulación de las entidades bancarias. Así la Reforma a la Ley de Bancos y Otras Instituciones Financieras reguló la inversión extranjera y nuevas formas de intermediación financiera como la banca universal y la banca de inversión, además de favorecer las fusiones entre diversas entidades bancarias (Bracho et al., 2002, p. 303).

Estas fusiones han modificado la estructura y composición del sistema financiero venezolano, ya que para el año 1994 existían I | 4 instituciones financieras (Bracho et al., 2002, p. 308) mientras que para diciembre de 2007 disminuyeron a 59 entidades, de las cuales 49 eran de capital privado y las 10 restantes pertenecian al Estado (SUDEBAN, 2007, p. 21).

Por otra parte, es importante destacar que para el año 1994 la participación más representativa del Sistema Bancario se encontraba en los subsistemas banca comercial y de inversión, agrupando el 55,3\% del total de entidades financieras existentes para la época, mientras que para diciembre del año 2007, los subsistemas banca universal (que no había en el año 1994) y comercial aglomeraron el 64,40\% del total de las instituciones bancarias legalmente autorizadas para funcionar en el territorio de la República Bolivariana de Venezuela.

Debido al auge de los precios del petróleo, durante el periodo 2004-2007, la tasa de crecimiento de la economía venezolana fue de las más altas entre las principales economías latinoamericanas y cercana a la de las economías emergentes más exitosas del planeta (Guerra y Olivo, 2009, p.4). Este auge fue compartido por el sector financiero Latinoamericano (Saza, 2010)

El impacto más importante de la crisis global sobre la economía venezolana se concretizó a partir de la caída de los precios petroleros que se inició desde julio del 2008 y se intensificó dos meses después a causa de la crisis bancaria de Estados Unidos y Europa (Guerra y Olivo, 2009, p. 7), considerada la peor crisis de los mercados financieros registrada desde la década de 1930 (Bravo, 2010, p. 3). Todo esto influyó para que el año 2009 la economía venezolana entrara en un ciclo recesivo (Vera, 20l0, p. 4) y se iniciara, a partir de febrero de ese año, una serie de intervenciones por parte del gobierno venezolano, a un grupo de instituciones financieras, que para febrero del año 20 I I sumaron, en total, 2 I entidades bancarias (SUDEBAN, 201 I).

El presente estudio abarcó el primer semestre de 2008. Para el cierre de ese lapso, la Banca Universal y la Comercial presentaron la mayor proporción del total de activo del sistema bancario venezolano, abarcando el 96,08\% de 246.300 millones de bolívares (SUDEBAN, 2008). Para esta fecha el dólar estaba regulado a 2, 15 bolívares por dólar.

\subsection{Grupos estratégicos}

El concepto de grupo estratégico de empresas se originó a partir de los años setenta con la investigación llevada a cabo por Hunt (1972), quien clasificó las empresas en cuatro grupos estratégicos e incluyó variables como la diversificación y diferenciación de los productos y el grado o magnitud de integración vertical dentro de la industria, para explicar la acción empresarial en una industria de productos genéricos.

Sin embargo, fue a partir de los trabajos de Porter (1979), Hatten (1974), Cool y Schendel (1987), entre otros, que el concepto de grupos estratégicos cobró relevancia.

En el ámbito de las dimensiones utilizadas en la presente investigación, Cool y Schendel (1987, p. I 106) definen a los grupos estratégicos como un conjunto de empresas que compiten en el interior de una industria y mantienen combinaciones similares de alcance y recursos comprometidos.

El análisis de grupos estratégicos busca identificar las empresas que tienen características estratégicas similares, que siguen estrategias parecidas o compiten sobre bases similares. En otras palabras, el propósito de llevar a cabo este tipo de análisis es determinar que características resultan más distintivas en un conjunto de organizaciones que pertenecen a un mismo sector productivo (Martínez y Milla, 2005, p. 53).

En ese orden de ideas, numerosas investigaciones se han llevado a cabo con el objeto de estudiar la relación entre la estrategia y el nivel de rentabilidad que obtienen las distintas empresas de un sector. Algunas de ellas han obtenido diferencias relevantes de desempeño entre los grupos estratégicos obtenidos, es decir, hallan la validez predictiva de los grupos estratégicos (McNamara et al., 2003). Mientras que 
otros investigadores niegan la validez predictiva de los grupos estratégicos (Houthoofd y Heene, 1997). Finalmente, investigadores han obtenido diferencias significativas en algunas de las variables de desempeño que analizan entre los grupos estratégicos obtenidos (Zúñiga et al., 2004).

Esta falta de consenso en los resultados obtenidos por diferentes investigaciones han provocado posiciones argumentadas a favor y en contra de la existencia de la validez predictiva de los grupos estratégicos que darán apoyo a las hipótesis alternativas que se pretender contrastar en esta investigación.

Así, autores como Cool y Schendel (1987) señalan que los grupos estratégicos son últiles por su validez predictiva, pero que la veracidad de dicha validez ha sido afectada por la existencia de importantes errores en los métodos empleados por algunos investigadores, tales como: I) Han utilizado medidas de desempeño a un nivel de agrupación empresarial diferente al que se ha empleado para identificar a los grupos; 2 ) Han utilizado medidas contables sin ajustar; 3) Se ha empleado una sola medida y no se han ajustado los resultados al riesgo soportado por la empresa.

Por otra parte, los dos enfoques de investigación de grupos estratégicos, el de la economía industrial y el de dirección estratégica, reconocen que existe una relación entre la estrategia adoptada por la organización y los resultados obtenidos por ella (Hitt et al., 2003, p. 65).

Según el primer enfoque el nivel de rentabilidad que logra una empresa está condicionado por el modelo Estructura-Conducta-Resultados, mientras que, de acuerdo a la perspectiva de la dirección estratégica, el desempeño de una firma depende de la ventaja competitiva derivada de la estrategia que ella adopta en función de sus recursos y capacidades, así como de su adaptación a las oportunidades y amenazas del entorno (Claver et al., 2006).

Al mismo tiempo, la existencia de barreras de movilidad entre los distintos grupos permiten que algunas empresas disfruten, de manera estable, de niveles de desempeño superiores a la media de su sector, porque las ventajas competitivas que obtienen con las estrategias implementadas serán difícilmente imitadas por las firmas restantes (Hitt et al., 2003, p. 65, Claver et al., 2006).

Otros argumentos a favor de la validez predictiva, se encuentran en los estudios de dinámica de grupos, que han concluido que las empresas encuentran mu- chas dificultades para cambiar de grupo (Claver et al., 2006, p. 25).

Por su lado, otros investigadores han concluido que no existen diferencias relevantes en los niveles de rentabilidad entre los distintos grupos estratégicos que conforman un sector $y$, por tanto, niegan la validez predictiva de los grupos estratégicos. Así Domowitz et al. (1987) compararon el comportamiento colusivo entre industrias fragmentadas $y$ concentradas, encontrando un nivel de colusión inferior al previsto y concluyeron que éste puede verse limitado por variados factores, tales como la elasticidad de la demanda, el poder de negociación de ciertos clientes y proveedores, la amenaza de nuevos competidores y de los ya existentes, que pueden modificar los planes de colusión.

En esa misma línea, el principio de equifinalidad (Mehra y Floyd, 1998) se opone a los argumentos de la validez predictiva de los grupos estratégicos, al considerar que distintas estrategias implantadas en un mismo sector pueden conducir al logro de niveles de rentabilidad similares.

Por su parte Zuñiga et al. (2004), consideran que una empresa puede decidir cambiar de grupo cuando su nivel de desempeño es bajo o sus posibilidades de supervivencia son bajas en comparación con las empresas de otros grupos.

Enfoques como el tradicional de la dirección estratégica de la empresa (Ansoff, 1965), el de la perspectiva de la elección estratégica (Child, 1997) y el de la perspectiva de la adaptación (Meyer y Rowan, 1977) ya habían desarrollado la postura de Zuñiga et al. (2004). Según estos tres enfoques, los niveles de desempeño de los distintos grupos son similares, bien porque los cambios en las estrategias de las empresas son rápidos, fáciles y sencillos o porque las empresas tratan de adaptarse al entorno.

Los autores de esta investigación adoptan las postura de Domowitz et al. (1987), Houthoofd y Heene (1997), Mehra y Floyd (1998), que niegan la validez predictiva de los grupos estratégicos. En todo caso, los resultados obtenidos en el presente estudio permitirán o no corroborar esta apreciación.

\subsection{Breves consideraciones sobre la cobertura y la exclusión geográfica financiera}

Las entidades bancarias procuran ubicar sus oficinas en aquellas regiones con mejores condiciones, tales 
como mayor desarrollo económico, mejor educación y con mayor dependencia de la economía formal, así que las zonas pobres tienden a ser excluidas del sistema financiero (Rojas, 2006, p. 16).

La exclusión geográfica financiera se refiere a aquellas regiones que no disponen de manera permanente de servicios bancarios formales, considerándose al sector financiero formal como aquellas entidades vigiladas por la Superintendencia Bancaria (Marulanda y Paredes, 2006).

La exclusión geográfica financiera es característica de las zonas rurales y de los estratos bajos de las franjas urbanas, lo que difículta y hace más costoso el acceso a los servicios financieros a las personas residenciadas en estas zonas, generando a su vez mayor desigualdad y menores posibilidades de desarrollo con respecto a los estratos medios o altos (Marulanda y Paredes, 2006).

Para algunos investigadores, esta exclusión puede ser una consecuencia del tamaño y ubicación de la red bancaria, que a su vez, depende, entre otros factores, de la misión, de la estrategia, de la población objetivo y de la actividad económica de la zona (Zahler, 2008, pp. 46,47). Por otra parte, el tamaño de la red también se relaciona con la calidad del servicio financiero (Flores, 2006, p. 25).

Entre las principales causas de inclusión y de exclusión geográfica financiera en Latinoamérica se tienen las siguientes (Zahler, 2008, pp. 12-14):

\subsubsection{Desarrollo económico y social}

Nivel de ingreso. Las regiones con bajo nivel de ingreso per cápita medio tienden a ser excluidas como mercado objetivo por las entidades bancarias, porque los individuos no tienen el ingreso mínimo que se requiere para ser sujeto de bancarización, razón por la cual sus formas de consumo bancario son más costosas y su nivel de riesgo es más elevado (Mendizabal et al., 2008, p. 2।4).

Educación. Las personas de ingresos medios y bajos, generalmente con menor educación y escaso conocimiento y práctica tecnológica necesaria para acceder a los servicios financieros, tienen dificultad para usar cajeros automáticos y tarjetas de crédito, e incluso temor a recurrir a la banca o simplemente una menor cultura financiera (autoexclusión), razones por las cuales no resultan un mercado interesante para las empresas financieras (Marulanda y Paredes, 2006, p. 8).
Informalidad. En la regiones donde los postulantes al crédito se encuentran en el mercado informal, donde existe una alta probabilidad de que se carezca de antecedentes para acreditar empleo, ventas, pagos de impuestos, entre otros, se elevan los riesgos a las entidades financieras si efectivamente llevan a cabo préstamos en esas condiciones.

\subsubsection{Organización industrial}

Concentración bancaria. Los bancos tienden a sesgar la oferta crediticia y demás servicios financieros hacia las regiones donde se concentran grandes empresas, donde no se afrontan los riesgos ni los altos costos de monitoreo que generan las pequeñas y medianas empresas.

Costos fijos. Las entidades bancarias tienden a no interesarse en captar clientes de medios y bajos ingresos ni a pequeñas y medianas empresas, porque los costos fijos de captación de estos segmentos, así como los de evaluación, desembolso, seguimiento y de recaudación de un crédito son mucho más elevados, y por tanto menos rentables. Por otro lado, las exigencias de parte de las instituciones financieras relativas a montos mínimos de depósitos y de préstamos así como a cobros de mantenimiento de cuentas desestimulan a los demandantes de servicios financieros (Mendizabal, et al., 2008, p. 213).

Riesgo de crédito de sectores de ingresos medios y bajos. Las empresas y personas asociadas a este nivel de ingresos tienen poca diversificación de sus fuentes de ingresos. Generalmente sus balances son poco transparentes, de allí que las tasas de interés deban ser más altas para compensar estos mayores riesgos a los que se enfrentan los acreedores. Pero en países como Venezuela existen limitaciones a las tasas máximas de interés que pueden cobrar las instituciones financieras formales, lo que conlleva a que este segmento tienda a quedar fuera de la bancarización.

Empresas de apoyo al giro. Las empresas que administran los cajeros automáticos, los puntos de venta y las tarjetas de crédito, tienden a sesgar la ubicación de estos equipos en negocios cuyos ingresos sean mayores que los costos de inversión y operación; lo que en la práctica, conlleva a la marginación de los segmentos de personas y empresas de ingresos y de ventas medios y bajos.

Costo de las sucursales. Los costos de instalación y operación de las oficinas bancarias son elevados y 
en las zonas con poca densidad poblacional no suele ser rentable, lo que se traduce en escasez de sucursales bancarias en lugares pequeños o poco poblados, marginando de la bancarización principalmente a los sectores rurales, típicamente de bajos ingresos.

\subsubsection{Regulación y normativa}

Tasas de interés máximas. En Venezuela existen límites superiores legales a las tasas de interés activas para proteger a los consumidores de prácticas usureras, pero en la realidad esa política trae como consecuencia que los sectores más riesgosos sean marginados del sistema financiero.

Esta inclusión y exclusión geográfica financiera tiene sus consecuencias. Así, un sistema financiero que funciona apropiadamente favorece el crecimiento económico, materializado, por ejemplo, en niveles más elevados del PIB per cápita y en la cohesión social; por el contrario, la exclusión geográfica financiera, dificulta el desarrollo económico y social, porque es esta industria la que precisamente promueve el ahorro y la inversión (Mendizabal et al., 2008, p. 214).

Por esta y otras razones, en países europeos una estrategia esencial de la lucha contra la exclusión social y la pobreza es la prevención de la exclusión financiera (Mendizabal, 2008, p. 213).

\subsection{Variables utilizadas en la presente investigación}

Para lograr el objetivo de esta investigación se consideraron únicamente las variables de la dimensión alcance de la estrategia para la conformación de los grupos estratégicos (Tabla I). Por otra parte, la variable A I, de la dimensión alcance de la estrategia, fue desglosada en 10 subvariables, 8 relacionadas con la especialización de la cartera de créditos según el sector productivo (Tabla 2) y las 2 restantes con el tipo de garantía utilizada para otorgar los préstamos (Tabla 3). Estas subvariables permitieron hacer un análisis más detallado, sobre la orientación de la cartera de créditos, de los grupos estratégicos obtenidos.

Finalmente, para evaluar el desempeño de los grupos se emplearon dos indicadores de resultados ( $\mathrm{Ta}$ bla 4) y para determinar la cobertura geográfica el número de municipios en los que se ubica, por lo menos, una oficina de un determinado grupo, así como la densidad poblacional de esos municipios para conocer el segmento de mercado al que se dirigen, desde este enfoque.

\section{Metodología}

\section{I. Muestra de entidades y fuentes de datos}

Para la conformación de grupos estratégicos en la banca venezolana se consideró el primer semestre

Tabla I

Variables de la dimensión alcance de la estrategia empleadas para conformar los grupos estratégicos en el sector bancario venezolano

\section{Variables}

Al Cartera de créditos/Inversiones financieras.

A2 Disponibilidades/Inversiones financieras.

A3 Cartera de valores/Inversiones financieras.

A4 Créditos a bancos/Activo.

A5 Cuentas corrientes/Pasivo

A6 (Ctas. ahorro + Depósitos a plazo)/Pasivo

A7 $\mathrm{N}^{\circ}$ de oficinas $/ \mathrm{N}^{\circ}$ de trabajadores.

A8 Activo total.
Descripción

Especialización en las economías domésticas y en la pequeña y mediana empresa. Refleja la banca comercial o al por menor.

Refleja una orientación conservadora y tradicional del activo.

Aproximación a la banca corporativa o de negocios.

Recursos destinados al mercado interbancario para la colocación de fondos.

Participación de instrumentos no tradicionales y costosos de captación en el pasivo.

Participación de los instrumentos tradicionales de captación en el pasivo.

Indicador del ámbito geográfico (territorialidad) de la estrategia bancaria. Mide la proximidad de los servicios bancarios a los clientes.

Tamaño de la entidad financiera 
Tabla 2

Variables de la especialización de la cartera de créditos según el sector productivo

\begin{tabular}{|c|c|c|}
\hline & Variables & Descripción \\
\hline Alind & CC Sector industrial/Cartera de Crédito & Especialización en el sector industrial \\
\hline Alagr & CC Sector agrícola/Cartera de Crédito & Especialización en el sector agrícola, agropecuario, pesquero y forestal \\
\hline Almin & $\begin{array}{l}\text { CC Sector minas e hidrocarburos/Car- } \\
\text { tera de Crédito }\end{array}$ & Especialización en el sector minas, canteras e hidrocarburos \\
\hline Alser & $\begin{array}{l}\text { CC Sector servicios públicos/Cart. Cré- } \\
\text { dito }\end{array}$ & $\begin{array}{l}\text { Especialización en el sector electricidad, gas, agua, transporte, almacenamiento y } \\
\text { comunicaciones }\end{array}$ \\
\hline Alcon & CC Sector construcción/Cart. Crédito & Especialización en el sector construcción \\
\hline Alcom & $\begin{array}{l}\text { CC Sector comercio/ Cartera de Cré- } \\
\text { dito }\end{array}$ & Especialización en el sector comercio mayor y detal, restaurantes y hoteles \\
\hline Alest & $\begin{array}{l}\text { CC Sector establecimientos financieros/ } \\
\text { Cartera de Crédito }\end{array}$ & $\begin{array}{l}\text { Especialización en el sector establecimientos financieros, seguros, bienes inmuebles } \\
\text { y servicios prestados a empresas }\end{array}$ \\
\hline Alcom & $\begin{array}{l}\text { CC Sector servicios comunales/ Car- } \\
\text { tera de Crédito }\end{array}$ & Especialización en el sector servicios comunales, sociales y personales \\
\hline
\end{tabular}

Fuente: Elaboración propia a partir de las notas a los estados financieros de las entidades bancarias.

Tabla 3

Variables de la garantía de la cartera de crédito

\begin{tabular}{lll}
\hline \multicolumn{2}{c}{ Variables } & \multicolumn{1}{c}{ Descripción } \\
\hline Al singarantía & CC sin garantía/Cart. Crédito & Especialización productiva en préstamos sin ningún tipo de garantía \\
Al garantíareal & CC con garantía real/Cart. & $\begin{array}{l}\text { Especialización en productiva en el mercado de préstamos hipotecarios y } \\
\text { Crédito }\end{array}$ \\
\hline
\end{tabular}

Fuente: Elaboración propia a partir de las notas a los estados financieros de las entidades bancarias.

Tabla 4

Variables de resultados

\begin{tabular}{lcl}
\hline \multicolumn{1}{c}{ Variables } & Notación & Definición \\
\hline Rentabilidad económica & REC & Resultado neto después de impuesto/Activo \\
Rentabilidad financiera & RFI & Resultado neto después de impuesto/Patrimonio \\
\hline
\end{tabular}

Fuente: Mas (1996, p. 89).

del año 2008 y, en principio, se incluyó la población total constituida por 60 bancos. Posteriormente, se excluyeron aquellos bancos que no publicaron sus estados financieros en ASOBANCA, ya que éstos, a diferencia de los que publica SUDEBAN, contienen las notas de los estados financieros consolidados, necesarias para evaluar la constitución de la cartera de crédito de las instituciones bancarias. Así, la muestra quedó conformada por 52 entida- des financieras, lo que representa el $86,67 \%$ del total de bancos que funcionaba para ese periodo ( $\mathrm{Ta}$ bla 5).

Se seleccionó como periodo de estudio el primer semestre del año 2008 por dos razones. En primer lugar porque, aunque la crisis financiera mundial se gestó a finales del primer semestre del año 2008 (Saza, 20l0), el auge económico se prolongó en va- 
Tabla 5

Composición de la muestra objeto de estudio para el primer semestre del año 2008

\begin{tabular}{|c|c|c|c|c|}
\hline Instituciones & Total & $\%$ & Muestra & $\%$ \\
\hline Bancos Universales & 24 & 40,00 & 22 & 36,67 \\
\hline Bancos Comerciales & 15 & 25,00 & 13 & 21,67 \\
\hline Bancos con Leyes Especiales & 4 & 6,67 & 2 & 3,33 \\
\hline Bancos de Desarrollo & 7 & 11,67 & 6 & 10,00 \\
\hline Bancos de Inversión & 4 & 6,67 & 3 & 5,00 \\
\hline Banco Hipotecario & । & 1,67 & । & 1,67 \\
\hline De Arrendamiento Financiero & I & 1,67 & । & 1,67 \\
\hline Entidades de Ahorro y Préstamo & 2 & 3,33 & 2 & 3,33 \\
\hline Fondos del Mercado Monetario & 2 & 3,33 & 2 & 3,33 \\
\hline Total & 60 & 100,00 & 52 & 86,67 \\
\hline
\end{tabular}

Fuente: SUDEBAN (2008, p. 43).

rios países de Latinoamérica durante ese semestre, produciéndose el quiebre de esa tendencia a mediados de septiembre, con el colapso de Lehman Brothers (Ocampo, 2009, p. 49). De manera que, el primer semestre del año 2008 se caracterizó, en Latinoamérica, por un ser un periodo de estabilidad económica antes de la crisis financiera mundial, y los autores de esta investigación estiman que el estudio debe efectuase considerando lapsos de tiempo antes, durante y después de la crisis para conocer posibles cambios en la estrategia financiera y de cobertura geográfica de las instituciones financieras venezolanas. Esta investigación, en una primera fase, abordó el período de tiempo previo a la crisis y, posteriormente, se estudiarán los períodos que corresponden a la manifestación de la crisis y a la atenuación de la misma.

Otra razón es que la Ley General de Bancos y Otras Instituciones Financieras (Artículo 194, N. ${ }^{\circ} 4$ ) obliga a las entidades bancarias a publicar sus estados financieros de manera semestral.

\subsection{Técnicas de procesamiento y análisis de datos}

Para determinar la relación entre las variables estratégicas se realizó un análisis de componentes principales (ACP), con rotación Varimax, como fase previa a la aplicación del análisis cluster (AC), con la finalidad de detectar valores atípicos y generar variables no correlacionadas entre sí (Gómez et al., 1997, p. $8 \mid 8$ ). Todas las variables fueron previamente transformadas en puntuaciones de la curva normal $Z$ ( $Z$ es $N(0, I)$ ) para estandarizar sus dimensiones, ya que algunas no seguían una distribución normal.
Por su parte, el AC permitió identificar y tipificar cinco grupos estratégicos. Esta metodología ha sido frecuentemente utilizada para la identificación de grupos estratégicos dada su idoneidad como sistema de clasificación, ya que agrupa empresas en conjuntos homogéneos internamente y heterogéneos entre sí (Flavian y Polo, 1999). En general, el análisis cluster ha sido empleado por Cool y Schendel (1987), Surroca (2003), Almanza y Daccarett (2005), entre otros.

Sin embargo, la identificación de los grupos a través del AC tiene dos inconvenientes a considerar (Flavian y Polo, 1999). Primero, que se trata de una técnica exploratoria que siempre permite la agrupación de empresas aunque tales agrupaciones no tengan sentido desde el punto de vista estratégico. Segundo, que engloba distintas variantes y algoritmos entre los cuales se puede optar, sin que ninguno de ellos se muestre definitivamente superior al resto. Afortunadamente estos inconvenientes pueden ser superados con determinados procedimientos, como el aplicado en esta investigación, que consiste en realizar un ACP previamente, para determinar la relación entre las variables seleccionadas y detectar valores atípicos.

Por otra parte, para distinguir las estrategias que caracterizaron a los diferentes grupos se siguió el procedimiento de uso común propuesto por Amel y Rhoades (1988), y para reforzar esta metodología se realizaron pruebas de constraste de medias a cada una de las variables de alcance de la estrategia. A las variables con distribución normal se les realizó la prueba de ANOVA y, posteriormente, se les aplicó la prueba de Tukey para determinar cuales grupos son estadísticamente diferentes, mientras que las res- 
tantes variables, que presentaron distribuciones no normales, se evaluaron con la Prueba de Kruskal-Wallis. La utilización de estas pruebas es novedoso en la identificación de las estrategias seguidas por los grupos estratégicos en el sector bancario y permite, a diferencia de los métodos comunmente utilizados, verificar la existencia de grupos estadísticamente diferentes y de grupos homogéneos, así como de categorías entre los grupos que desarrollan una misma estrategia.

Por otro lado, con el objetivo de determinar las diferencias de desempeño entre los grupos estratégicos obtenidos, a las variables rentabilidad económica y financiera, se les aplicó las pruebas de ANOVA y Kruskal-Wallis, respectivamente, porque presentaban las mismas condiciones que las variables de alcance de la estrategia. Sirvió para verificar la hipótesis de relación estrategia y resultados.

Para la tabulación de los datos y para aplicar todos los análisis y las pruebas descritas anteriormente se empleó el paquete estadístico SPSS (versión 15.0). Mientras que para evaluar la cobertura geográfica de la banca y evidenciar la relación entre ésta y los grupos estratégicos se utilizó el software de sistema de información geográfica Arcgis (versión 9.2). Finalmente, se empleo la prueba Rho de Spearman (rs) para analizar la competencia entre los grupos estratégicos y su cobertura en los municipios menos pobres y en los más atractivos para invertir.

\section{Resultados}

\section{I. Relaciones entre las variables estratégicas (Componentes principales)}

Para lograr este objetivo específico se introdujo una modificación en la metodología propuesta por Mas (1994) y Almanza y Daccarett (2005) para detectar grupos estratégicos, ya que se realizó un análisis de componentes principales (ACP) como fase previa a la aplicación del análisis cluster (AC), con la finalidad de detectar valores atípicos, factores estratégicos $y$, más importante aún, generar variables no correlacionadas entre sí, porque el AC es sensible a la multicolinealidad, problema que puede ser superado con procedimientos como el descrito (Pérez, 2005, p. 442).

Debido a que tres de las siete variables analizadas no siguen una distribución normal, todas las variables fueron transformadas en puntuaciones de la curva normal $Z(Z \sim N(0,1))$ para estandarizar sus dimensiones (Cáceres, 2007, p. 2 I6). Luego, se aplicó el ACP utilizando la matriz de coeficientes de correlaciones entre variables (tipo R), para la obtención de los autovalores y autovectores, que arrojó una matriz de correlaciones no definida positiva, cuyo determinante fue cero (Carrasco, 2009, p. 9), debido a la multicolinealidad negativa aproximada entre las variables A I y A3 ( $r=-0,93)$, lo cual sugiere que, al menos para los datos y el momento analizado, el comportamiento de una de estas variables es opuesto al de la otra, es decir, que a medida que un banco destina mayor cantidad de dinero a su cartera de créditos (AI) dispone de menos recursos para su cartera de valores (A3), y viceversa. Esta información es redundante, ya que la suma de $\mathrm{Al}, \mathrm{A} 2$ y $\mathrm{A} 3$ es igual a uno, por lo que fue necesario excluir $A 3$ para formar los grupos estratégicos y evitar problemas en el desarrollo de algoritmos de cálculos (Ketchen y Shook, 1996), aunque fue utilizada, de forma suplementaria, en análisis posteriores.

En una segunda corrida de los datos, un banco (Participaciones Vencred, S.A.) se separó de los demás al correlacionar a los individuos con los dos primeros componentes principales. Al descartar este atípico, tal como sugieren Gual y Hernández ( 99 |, p. 706), se estandarizó de nuevo la data y se realizó la última corrida. Los tres primeros están asociados a autovalores mayores que la unidad, pero se agregó un cuarto componente inferior a la unidad, para aplicar el criterio de retener los componentes que sumen más de 80\% de varianza total (Peña, 2002, p. I55).

Para interpretar el significado de los componentes (o factores) se consideran las correlaciones iguales o mayores a 0,5 (en valores absolutos) de las variables con al menos uno de los componentes (Mongay, 2005, p. 196). El primer componente presenta altas correlaciones con las variables A5, A2 y, en menor grado, con la variable Activo, que por tener mayor saturación en el tercer componente no es muy apropiada para interpretar el primero de ellos (Tabla 6). Debido a que las correlaciones son positivas, se infiere que en la medida que los bancos presentan mayor ratio de Cuentas corrientes/Pasivo también presentan mayores Disponibilidades/Inversiones Financieras, lo cual indica que al aumentar la participación en el pasivo de instrumentos no tradicionales y costosos se incrementa la disponibilidades en caja, banco y efectos de cobro inmediato, es decir, se incrementa la orientación conservadora y tradicional del activo para eludir riesgos e incurrir en costos operativos menores. Este primer componen- 
te es el más importante, pues refleja que la mayor variabilidad (o donde hay mayor competencia bancaria) en la adopción de las estrategias ocurre en A5 y A2, por lo cual se puede definir este primer componente como factor de estrategia de activo conservador y pasivo innovador.

El segundo factor (componente) estratégico se correlaciona positivamente con $\mathrm{Al}$ y negativamente con $A 4$ y, también, con $A 7$, que presenta su mayor saturación en el cuarto componente, por lo cual no es muy relevante para interpretar este segundo factor. Es importante destacar que la variable A3, eliminada por su multicolinealidad negativa casi exacta con AI, presenta un comportamiento predecible a partir de esta última variable. Estas relaciones ocurren porque a medida que los Bancos enfatizan la estrategia de incrementar la Cartera de crédito/Inversiones Financieras, disminuyen los Créditos a bancos/Activo $y$, principalmente, las colocaciones en la cartera de valores. Este componente se puede denominar factor de estrategia de activo innovador.

El tercer factor (componente) estratégico se correlaciona positivamente con el activo y negativamente con A6, que está mejor representada por su mayor nivel de saturación $(-0,63)$ y por no tener saturaciones significativas en los demás componentes. Se deduce entonces que, para un grupo de bancos, a medida que tienen mayor tamaño, el ratio (Cuentas de ahorro + Depósitos a plazo)/Pasivo es menor. Este componente se denomina factor de estrategia de gran tamaño y pasivo no conservador.

Finalmente, el cuarto factor (componente) estratégico se correlaciona positivamente con A7 (que tiene una saturación menor en el componente 2). Este componente se designa como factor de estrategia de territorialidad.

En sintesis, cada factor refleja un patrón distinto de conducta estratégica de los Bancos y las variables que saturan en un único factor determinan esa conducta. Por el contrario, las variables que saturan en más de un factor indican que son relevantes en más de un patrón de conducta estratégica (Ruiz et al., 1999, p. 104).

\subsection{Identificación y caracterización de los grupos estratégicos}

Con los cuatro primeros componentes principales se realizó un análisis clúster jerárquico, cuyo dendograma, permitió establecer un total de cinco grupos estratégicos (GE), que resultan los menos dispares en número de elementos entre todos los grupos posibles (Tabla 7).

\subsubsection{Caracterización de los grupos estratégicos}

Para identificar la estrategia que distingue a un grupo de otro se compararon dos procedimientos. En

Tabla 6

Correlaciones ( $r$ ) de las variables con los 4 primeros componentes principales

\begin{tabular}{|c|c|c|c|c|}
\hline \multirow{2}{*}{ Variables } & \multicolumn{4}{|c|}{ Componentes } \\
\hline & 1 & 2 & 3 & 4 \\
\hline Cuentas corrientes/Pasivo (A5) & 0,85 & 0,04 & 0,23 & $-0,04$ \\
\hline Disponibilidades/Inv. Financiera (A2) & 0,82 & $-0,14$ & $-0,01$ & 0,43 \\
\hline Activo & 0,56 & 0,14 & 0,63 & $-0,12$ \\
\hline (C/ahorro+depósitos a plazo)/Pasivo (A6) & 0,43 & 0,35 & $-0,68$ & 0,28 \\
\hline Créditos a bancos/Activo (A4) & 0,29 & $-0,73$ & $-0,28$ & $-0,38$ \\
\hline Cartera de crédito/Inv. Financiera (A I) & $-0,21$ & 0,85 & 0,11 & $-0,01$ \\
\hline N. ${ }^{\circ}$ Oficinas $/ N{ }^{\circ}$ de trabajadores (A7) & $-0,47$ & $-0,50$ & 0,31 & 0,57 \\
\hline Varianza explicada por componente (\%) & 32,01 & 23,99 & 15,74 & 10,82 \\
\hline Varianza total explicada (\%) & \multicolumn{4}{|c|}{82,57} \\
\hline $\begin{array}{l}\text { Determinante } \approx 0,000 \\
\text { Prueba de esfericidad de Bartlett Chi-cuadr } \\
\text { Medida de adecuación muestral de Kaiser-M }\end{array}$ & & & & \\
\hline
\end{tabular}

Fuente: Cálculos propios. 
Tabla 7

Composición de los grupos estratégicos en el primer semestre del año 2008

\begin{tabular}{|c|c|c|c|c|}
\hline GEI & GE2 & GE3 & GE4 & GE5 \\
\hline $\begin{array}{c}\text { Caribe, Exterior, BNC, BFC, Canarias, } \\
\text { Venezolano de Crédito, Corpbanca, } \\
\text { Caroní, Bancoro, Provivienda, Central, } \\
\text { Del Sur, Sofitasa, Totalbank, Federal, } \\
\text { Bolívar, Confederado, Guayana, Plaza, } \\
\text { I00\% Banco, Stanford, Banvalor, } \\
\text { Bannorte, Activo, Real, Sofioccidente, } \\
\text { Mi Casa, Casa Propia }\end{array}$ & $\begin{array}{c}\text { Inverunión, } \\
\text { Comercio Exterior, } \\
\text { Bangente, Mi Banco, } \\
\text { Del Sol, Bancrecer, } \\
\text { Bancamiga, } \\
\text { Baninvest, Anfico }\end{array}$ & $\begin{array}{c}\text { Banesco, Mercantil, } \\
\text { Venezuela, BBVA, } \\
\text { BOD }\end{array}$ & $\begin{array}{c}\text { Citibank, } \\
\text { Banfoandes, Del } \\
\text { Tesoro, Abn Amro, } \\
\text { Helm, BIV }\end{array}$ & $\begin{array}{c}\text { Federal Banco de } \\
\text { Inversión, } \\
\text { Inverbanco, Federal } \\
\text { Fondos del Mercado } \\
\text { Monetario }\end{array}$ \\
\hline
\end{tabular}

Fuente: Elaboración propia.

primer lugar se empleó el propuesto por Amel y Rhoades (1988) y utilizado por Mas (1994, 1996) y Gómez et al., (1997), que consiste en calcular los valores medios de las variables estratégicas para cada grupo; luego, se obtienen los valores medios de los indicadores para la industria bancaria y, finalmente, se comparan los ratios de cada grupo con el promedio sectorial, concluyendo que un grupo estratégico se especializa en una determinada dimensión cuando el valor del ratio que la especifica es un 5\% superior al coeficiente medio de la industria, mientras que si el valor es 5\% inferior indicaría que el grupo intenta evadir la dimensión en cuestión.

El segundo procedimiento consistió en realizar pruebas de contraste de medias a cada una de las variables utilizadas para conformar los grupos estratégicos, lo cual demostró la existencia de diferencias significativas entre los grupos en términos de las variables de alcance de la estrategia confirmando así la validez de los cluster (Garcés, 2007, p. I55), ya que al menos uno de los GE presentó una media diferente en cada una de las variables estratégicas. Es importante destacar que a las variables con distribución normal ( $A$ I , A3, A5, A6 y A7) se les realizó la prueba de ANOVA y, posteriormente, se les aplicó la prueba de Tukey (prueba post hoc) para determinar cuáles grupos son estadísticamente diferentes. Las restantes variables se evaluaron con la Prueba de Kruskal-Wallis por presentar distribuciones no normales y, luego, se utilizó esta misma prueba, tomando los GE por pares, como prueba post hoc. Esto se realizó con la finalidad de reforzar la metodología propuesta por Amel y Rhoades (1988), identificando posibles gradaciones en el comportamiento de los grupos estratégicos con respecto a una variable particular. Por ejemplo, entre los grupos que adoptan una estrategia puede haber diferencias en la intensidad con que asumen dicha estrategia. Obviamente estas posibles diferencias se hacen relevantes en la medida que aumenta el tamaño de los clúster debido a la mayor estabilidad de las varianzas intragrupales. Estas gradaciones en la aplicación de las estrategias no pueden ser detectadas con el método de Amel y Rhoades ( 1988), de allí lo importante del procedimiento propuesto, porque permite lograr una mayor precisión estadística en los resultados, que a vez, implica un mejor análisis de las variables de alcance de la estrategia empleadas por los diferentes grupos.

En la Tabla 8 se presentan los dos procedimientos para identificar las estrategias adoptadas por los GE, a fin de comparar sus resultados. En esa Tabla se evidencia que ambas metodologías permiten identificar la estrategia que distingue a un grupo de otro, coincidiendo en las dimensiones en las que se especializa el GE y, de la misma manera, en las dimensiones que intentan evadir los GE, salvo en el GE5 en lo que respecta a la variable A4 (Créditos a bancos/Activo), ya que según el procedimiento propuesto por Amel y Rhoades (1988) este GE se especializa en dicha estrategia, porque el valor del ratio es un $5 \%$ superior al coeficiente medio de la industria, mientras que al efectuar los contrastes de medias, el GE5 es indiferente $(p<0,05)$ a la estrategia de otorgar créditos a otras instituciones financieras (A4).

Con respecto a la variable activo, en la Tabla 8 , se demuestra que el GE3 y el GE4 están constituidos por bancos de gran tamaño.

En función de sus características diferenciales obtenidas por los procedimientos de Amel y Rhoades ( 1988) y contrastes de medias, los grupos estratégicos antes identificados fueron etiquetados de la manera siguiente:

Grupo Estratégico I (GEI): Banca de tamaño moderado y muy conservadora con respecto al pasivo y al ac- 
Tabla 8

Caracterización de los grupos estratégicos (promedios de los valores de las variables de alcance) según procedimientos de Amel y Rhoades (1988) y contrastes de medias

\begin{tabular}{|c|c|c|c|c|c|c|c|c|}
\hline \multirow{2}{*}{ Variables estratégicas } & \multicolumn{5}{|c|}{ GE } & \multirow{2}{*}{ Sector } & \multirow{2}{*}{$\bar{x}-5 \%$} & \multirow{2}{*}{$\bar{x}+5 \%$} \\
\hline & 1 & 2 & 3 & 4 & 5 & & & \\
\hline Cartera de crédito/Inv. Financieras & $\underline{0,52 b}$ & $\underline{0,60 \mathrm{~b}}$ & $\underline{0,56 b}$ & $0,24 a$ & $0,33 a b$ & 0,50 & 0,47 & 0,52 \\
\hline Disponibilidades/Inv. Financieras & $\underline{0,24 b}$ & $0, \mathrm{II} \mathrm{I}$ & $\underline{0,25 b}$ & $\underline{0,25 b}$ & $0,16 a b$ & 0,21 & 0,20 & 0,22 \\
\hline Cartera de valores/Inv. Financieras & $0,24 a$ & $0,29 a b$ & $0,19 a$ & $\underline{0,5 \mathrm{Ib}}$ & $\underline{0,5 \mathrm{Ib}}$ & 0,29 & 0,28 & 0,31 \\
\hline Créditos a bancos/Activo (A4) & $0,06 a$ & $0,05 a$ & $0,05 a$ & $\underline{0,32 b}$ & $\underline{0,13} a b$ & 0,09 & 0,09 & 0,10 \\
\hline Cuentas corrientes/Pasivo (A5) & $\underline{0,30 b}$ & $0,04 a$ & $\underline{0,47 b}$ & $\underline{0,5 \mathrm{Ib}}$ & $0,00 \mathrm{a}$ & 0,28 & 0,27 & 0,29 \\
\hline (C/ahorro+depósitos a plazo)/Pasivo & $0,37 b$ & $0,18 a b$ & $0,25 a b$ & $0,25 a b$ & $0,00 \mathrm{a}$ & 0,29 & 0,27 & 0,30 \\
\hline $\mathrm{N} .^{\circ}$ Oficinas $/ \mathrm{N} .^{\circ}$ de trabajadores & $0,08 a$ & $0,03 a$ & $0,05 a$ & $0,03 a$ & $\underline{1,00 \mathrm{~b}}$ & 0,11 & 0,11 & 0,12 \\
\hline Activos (MM) & 3.289 & 303 & $\underline{23.460}$ & $\underline{5.418}$ & 635 & 4.834 & 4.592 & 5.076 \\
\hline
\end{tabular}

: Estrategia diferenciada según procedimiento de Amel y Rhoades.

a, b: Diferencias entre pares de grupos según prueba de Kruskal-Wallis (variables A2 y A4) y prueba de Tukey (resto de variables).

ng: No se utilizó en la formación de los grupos estratégicos por presentar multicolinealidad.

Fuente: Elaboración propia.

tivo. Su principal estrategia con relación al pasivo es obtener recursos a través de instrumentos tradicionales, estables y económicos como las cuentas de ahorro y los depósitos a plazo (A6). Así que el GEI se especializa en las operaciones de banca al por menor, basadas en la captación y estímulo del ahorro familiar.

Con respecto al activo, también son muy conservadores, ya que buscan incurrir en costos operativos menores y eludir el riesgo al mantener sus fondos en las disponibilidades (A2) constituidas por caja, bancos y efectos de cobro inmediato.

En lo que respecta a la cartera de créditos, en promedio, el 32\% de los préstamos está respaldado con garantía real y el 17\% no tiene ningún tipo de garantía. El resto de la cartera de créditos está amparado con garantías personales.

Este grupo es el más numeroso (28 bancos) y representa el $46,67 \%$ de la muestra.

Grupo Estratégico 2 (GE2): Banca (comercial) muy pequeña especializada en economías domésticas y en la pequeña y mediana empresa. Está orientado, de manera clara y con mayor agresividad que el resto de los grupos, a la colocación de sus fondos en la cartera de créditos ( $\mathrm{Al}$ ), especialmente a los sectores comercio; industria y establecimientos financieros, seguros y servicios a empresas; con una fuerte tendencia hacia los microcréditos a empresarios, de ma- nera que se trata de una banca comercial. En promedio el $20 \%$ de sus créditos está respaldado con garantía real y sólo el 5\% no tiene ningún tipo de garantía. El 75\% restante está respaldado con garantías personales. Por ello, se infiere que representa una banca conservadora con respecto a la gestión de su cartera de crédito, lo que la prevendría del deterioro en situaciones de crisis económicas (Aristimuño y Asociados, 2005).

Grupo Estratégico 3 (GE3): Banca minorista de gran tamaño con instrumentos costosos de captación de pasivos. Capta sus pasivos, principalmente, a través de las cuentas corrientes (A5), que constituyen una política mucho más costosa que las cuentas de ahorro y los depósitos a plazo, pues involucra un mayor número de transacciones. En lo referente al activo, combinan una estrategia muy conservadora, barata y segura como las disponibilidades en caja, banco y efectos de cobro inmediato (A2) con otra más riesgosa de colocación de fondos como la cartera de créditos (AI).

Es el más arriesgado en la gestión de su cartera de crédito, ya que, en promedio, el $46 \%$ de sus préstamos no tiene ningún tipo de garantía, lo que puede conllevar al rápido deterioro de esta cartera y, en consecuencia, al detrimento en la calidad de sus activos si se agrava la situación económica del país (Solís et al., 2000; Aristimuño y asociados, 2005). En un escenario de crisis económica, la falta de garantía puede vulnerar gran parte del sistema bancario venezolano, tal 
como ocurrió en México en el año 1994 (Solís et al., 2000; Correa y Girón, 2004), ya que este GE está compuesto por las cinco entidades más grandes del país.

Este grupo tiene la mayor red de oficinas y cajeros automáticos en el territorio nacional, constituida, en promedio, por 308 sucursales y 502 cajeros, lo que la convierte en banca minorista, ya que un número elevado de oficinas es indicador de orientación a este segmento (Mas, 1994, p. 102).

\section{Grupo Estratégico 4 (GE4): Banca pública corporativa.} Las entidades financieras que forman parte de este grupo, tres pequeños bancos privados y los tres bancos públicos más importantes del país, están orientadas al segmento corporativo (A3), donde las operaciones overnight (A4) son una estratégia fundamental que las distingue del resto de los grupos. La magnitud de la cartera de valores en la banca pública se debe, entre otros factores, a que tiene más del $50 \%$ de su activo en inversiones en títulos valores del sector privado y en bonos de Deuda Pública Nacional (Tejero, 2007).

También tiene una estrategia conservadora del activo, aunque menos relevante que la anterior, al mantener en elevados niveles las disponibilidades (A2). Por su parte, el pasivo proviene primordialmente de las cuentas corrientes (A5).

Este grupo tiene una red moderada de oficinas y cajeros constituida, en promedio, por 60 sucursales y 60 cajeros. Esta redes pertenecen en su mayoría a los bancos públicos, pues los bancos privados que también conforman el GE4 tienen, en promedio, menos de cuatro sucursales y no poseen cajeros.

Grupo Estratégico 5 (GE5): Banca pequeña corporativa. Orientada principalmente a colocar sus fondos en la cartera de valores (A3), donde, según el procedimiento de Amel y Rhoades (1988), destacan los créditos a otras instituciones financieras (A4). Sin embargo, el procedimiento de contrastes de medias re- vela que este grupo es indiferente a la estrategia de otorgar créditos a otras instituciones financieras (A4).

Cada banco de este GE tiene una oficina en el país y es el grupo menos numeroso (3 bancos), que representan el $5 \%$ de la muestra.

\subsection{Diferencias de desempeño entre los grupos estratégicos}

Los grupos identificados anteriormente utilizan distintas estrategias para competir, ahora bien es importante determinar si éstas influyen en el desempeño. Es decir, si existen discrepancias de resultados entre los grupos estratégicos con relación a las medidas de resultados de rentabilidad económica y rentabilidad financiera $\left(\mathrm{H}_{1}\right)$.

Para comparar las diferencias de desempeño entre los grupos estratégicos, se estimaron las rentabilidades semestrales tanto económicas como financieras de cada banco para el periodo estudiado, clasificándolos de acuerdo al grupo estratégico que conforman, para evaluar si la la rentabilidad (variable dependiente) se relaciona con el grupo estratégico (variable independiente). Posteriomente se procedió a realizar la comparaciones de los valores promedios o de las medianas de las rentabilidades de los grupos, dependiendo si se cumplian o no los supuestos del modelo.

Dado que la variable rentabilidad económica presenta una distribución normal se utilizó la prueba de Anova, supuesto que no se cumplió para la rentabilidad financiera y, por ello, se empleó la prueba de Kruskal-Wallis, que compara las medianas (Tabla 9). Los resultados indican que no hay diferencias estadísticas entre las rentabilidades económicas obtenidas por los diferentes grupos estratégicos comparados $(p<0,05)$, aunque hubo variación de las mismas entre $0,00947(0,947 \%)$ y 0,01955 (I,955\%). Para el caso de la rentabilidad financiera, existen diferencias estadísticas si se eleva el margen de error a 10\%

Tabla 9

Contraste de medias para las variables de resultados de los grupos estratégicos obtenidos

\begin{tabular}{lcccccccc}
\hline \multirow{2}{*}{ Rentabilidad semestral (\%) } & \multicolumn{9}{c}{ Grupos } & Sig. & Tipo de Prueba \\
\cline { 2 - 6 } & GI & G2 & G3 & G4 & G5 & & ANOVA \\
\hline Económica (media) & 0,95 & 1,07 & 1,45 & 1,64 & 1,96 & 0,785 & AN \\
Financiera (mediana) & 12,13 & 3,16 & 17,31 & 14,60 & 1,62 & 0,092 & Kruskal-Wallis \\
\hline
\end{tabular}

Fuente: Elaboración propia a partir de los datos extraídos de los estados financieros de los bancos. 
$(p<0,10)$, destacando que el grupo 2 presentó un valor negativo cuando se estimó su promedio de rentabilidad financiera. De acuerdo a estos resultados se acepta la hipótesis nula $\left(\mathrm{H}_{0}\right)$.

Estos resultados coinciden con los obtenidos por Garcés y Duque (2008), quienes no encontraron evidencias de la relación entre las diferencias en el desempeño y la pertenencia al grupo estratégico. Igualmente, autores como Domowitz et al. (1987), Houthoofd y Heene (1997), Mehra y Floyd (1998) niegan la validez predictiva de los grupos estratégicos.

\subsection{Relación entre la cobertura geográfica y los grupos estratégicos}

Para lograr este objetivo, las ubicaciones espaciales de todas las oficinas de cada GE fueron superpuestas en un SIG que contenia información sobre limites geográficos y otras variables a nivel de municipios y estados de Venezuela, con el propósito de detectar posibles patrones de distribución espacial (cobertura geográfica) de los GE. Además, se empleo la prueba Rho de Spearman ( $r s$ ) para analizar la competencia entre los grupos estratégicos y su cobertura en los municipios clasificados según su grado de atractivo para las inversiones.

En detalle, para determinar la cobertura geográfica se consideró el número de municipios en los que se ubica, por lo menos, una oficina de un determinado GE, así como la densidad poblacional, atractivo para invertir y ranking de pobreza de esos municipios, para conocer el segmento de mercado al que se dirigen (Roche et al., 2002; CEPAL-UNICEF, 2006; CONAPRI, 2007). Así, por ejemplo, los bancos que integran el GEI tienen oficinas en 200 municipios de los 335 que conforman el territorio venezolano (Anexo I), de manera, que este grupo tiene una cobertura geográfica del $59,70 \%$. El GEI tiene en total I.7I 6 oficinas, de las cuales 905 se ubican en los municipios menos pobres de Venezuela y en los más atractivos para invertir, concentrando el $52,74 \%$ de sus oficinas en esos municipios (Anexosl, 2 y 3 ).

\subsection{GEI: Banca de tamaño moderado y muy conservadora con respecto al activo y al pasivo}

El GEI tiene una cobertura geográfica del 59,70\% (Anexo I), que es la más elevada de los cinco grupos estratégicos y, concentra el 52,74\% del total de sus oficinas en los municipios menos pobres de Venezuela y en los más atractivos para invertir (Anexos 2 y 3 ).
Esto demuestra que parte de la estrategia de cobertura de este grupo estratégico es posicionarse de manera agresiva en aquellos municipios con mayor desarrollo económico. Se trata de una banca no sólo conservadora con respecto al activo y al pasivo, sino también con relación al mercado geográfico al que va dirigido, ya que al situarse en estas zonas no afronta los riesgos ni los altos costos de monitoreo que se generan en aquellas regiones poco pobladas o con bajo nivel de ingreso. El GEI busca captar clientes con mayores ingresos y, por tanto, más rentables.

\subsubsection{GE2: Banca (comercial) muy pequeña especializada en economías domésticas y en la pequeña y mediana empresa}

Tiene una cobertura geográfica del 4, I8\% (Anexo I), y se tiende a ubicar en las capitales de los estados más desarrollados del país. Incluye financieramente a tres de los municipios menos pobres de Venezuela y siete de los más atractivos para invertir, estableciendo un $81,82 \%$ de sus oficinas en estos municipios (Anexos 2 y 3 ), especialmente en los más atractivos para invertir, donde a medida que el municipio es más atractivo tiende a tener mayor número de oficinas $(p<0,05)$, lo que evidencia una estrategia de cobertura enfocada en los mejores mercados geográficos.

Esta estrategia de cobertura puede deberse, entre otras, a las razones siguientes: A) Que se trata de regiones donde hay mayor número de personas integradas a la economía formal, por tanto, al otorgárseles los créditos hay menores riesgos con respecto a las zonas más pobres. B) Se trata de zonas donde la banca, en general, concede el mayor volumen de créditos a microempresarios (Melandri, 2006), lo cual es fundamental para su estrategia de banca comercial, especializada en la oferta de productos y servicios a la microempresa.

\subsubsection{GE3: Banca minorista de gran tamaño con instrumentos costosos de captación de pasivos}

Ocupa el tercer lugar en cobertura geográfica $(52,54 \%)$ y centraliza el $54,41 \%$ del total de sus oficinas en los municipios menos pobres de Venezuela y en los más atractivos para invertir (Anexos 1, 2 y 3).

En lo que respecta a la competencia para el GE3, desde el punto de vista geográfico, el GEI es su mayor 
contendiente, ya que ambos ubican al menos una oficina en 140 municipios, evidenciándose la fuerte rivalidad entre ambos grupos por esos mercados geográficos. Tanto en los municipios menos pobres como en los más atractivos para invertir, el GE3 está correlacionado positivamente $(p<0,05)$ con el GEI en número de oficinas (cobertura), de modo que ambos grupos buscan agresivamente posicionarse en los mejores mercados geográficos.

\subsubsection{GE4: Banca pública corporativa}

Tiene una cobertura geográfica del 57,01\% (Anexo I), que es la segunda más elevada de todos los grupos estratégicos. Se ubica, con respecto a los otros grupos estratégicos, en zonas menos pobladas y, aunque también incluye financieramente a siete de los municipios menos pobres de Venezuela y a los más atractivos para invertir, no lo hace de manera agresiva, ya que sólo concentra en ellos un 34,44\% de sus sucursales (Anexos 2 y 3). Esto demuestra que la estrategia de cobertura geográfica del GE4 no es concentrar sus oficinas en los municipios con mayor desarrollo económico sino distribuirlas en varios municipios, incluidos algunos de los más despoblados y los más pobres (Anexos 4 y 5), evidenciando el enfoque social de la banca pública, que prioriza a la rentabilidad social sobre la rentabilidad lucrativa.

\subsubsection{GE5: Banca pequeña corporativa}

Cada banco que integra este grupo tiene una sola oficina en Venezuela, ubicadas en el municipio Chacao estado Miranda y, por ello, tiene una cobertura geográfica de apenas 0,30\% (Anexo I), que es la más baja de todos los grupos estratégicos comparados.

Sin embargo, considerando que este grupo tiene como objetivo colocar sus fondos en la cartera de valores (A3) no necesita expandirse geográficamente para llevar a cabo esta estrategia. Pero si es importante su ubicación en el municipio Chacao, ya que éste colinda con el municipio Libertador (Caracas), lugar donde se efectúan operaciones financieras con títulos valores y donde se asientan la mayoría de las sedes principales de los bancos que integran los otros grupos estratégicos, si lo que se pretende es otorgar créditos a otras instituciones financieras (A4).

\section{Conclusiones}

La selección de las variables que representan el alcance de la estrategia evidenció que los bancos si- guen patrones de conducta estratégica diferentes. Así se obtuvieron cuatro componentes, el primer de ellos definido como factor de estrategia de activo conservador y pasivo innovador refleja que los bancos, a medida que aumentan la participación en el pasivo de las cuentas corrientes incrementan la disponibilidades en caja, banco y efectos de cobro inmediato. El segundo componente denominado factor de estrategia de activo innovador indica que a medida que los bancos incrementan su cartera de crédito disminuyen las colocaciones en la cartera de valores y los Créditos a bancos. El tercer componente denominado factor de estrategia de gran tamaño y pasivo no conservador, señala que los bancos a medida que son más grandes tienden a no captar pasivos a través de las cuentas de ahorro y los depósitos a plazo. El cuarto componente se relaciona con la estrategia de territorialidad.

Los grupos estratégicos combinan patrones de conductas. En este trabajo se obtuvieron cinco grupos estratégicos: Banca conservadora (GEl): Capta recursos, principalmente, a través de las cuentas de ahorro y depósitos a plazo y presenta una estrategia conservadora del activo; Banca comercial (GE2): Coloca sus fondos en la cartera de créditos, otorgando préstamos principalmente a microempresarios; Banca minorista (GE3): Banca de gran tamaño que capta su pasivo a través de las cuentas corrientes, mientras que su principales estrategias de activo son las disponibilidades y la cartera de créditos, donde el $46 \%$ de los préstamos no tiene ningún tipo de garantía; Banca pública corporativa (GE4): Orientada al segmento corporativo, con un activo constituido en más del 50\% por inversiones en títulos valores del sector privado y en bonos de Deuda Pública Nacional y Banca pequeña corporativa (GE5): Dirigida a colocar sus fondos en la cartera de valores, que incluye otorgar créditos a otras instituciones financieras.

Las diferentes estrategias adoptadas por los bancos no se expresaron en una diferenciación en los niveles de desempeño económico y financiero, coincidiendo estos resultados con los obtenidos por Garcés y Duque (2008), y en concordancia con la postura de autores como Domowitz et al. (1987), Houthoofd y Heene (1997), Mehra y Floyd (1998) que niegan la validez predictiva de los grupos estratégicos.

Los grupos bancarios llevan a cabo una estrategia de cobertura geográfica relacionada con la estrategia financiera. Así la Banca conservadora concentra sus ofi- 
cinas en los municipios menos pobres y en los más atractivos para invertir, que son menos riesgosos y más rentables. La Banca comercial especializada en la oferta de productos y servicios a la microempresa, ubica el $81,82 \%$ de sus oficinas en aquellos municipios donde la banca, en general, otorga el mayor volumen de créditos a microempresarios. Banca minorista: Posee la red más grande de oficinas y tiene una cobertura geográfica del 52,54\%, compitiendo en este aspecto con el GEI. Banca pública corporativa: Tiene una red moderada de oficinas que incluye algunos de los municipios menos pobres, pero esta cobertura no la hace de manera agresiva. También se sitúa en los municipios más pobres, lo que evidencia una mayor responsabilidad social, acorde con la misión de las empresas públicas. Banca pequeña corporativa: Se ubica en Chacao, cerca de la mayoría de las sedes de los bancos que integran los otros grupos estratégicos, lo que le facilita su estrategia de colocar sus fondos en la cartera de valores, que incluye otorgar créditos a otras instituciones financieras.

\section{Bibliografía}

AMEL, D. y RHOADES, S. ( 1 988), «Strategic groups in banking», Review of Economics and Statistics, Vol. 70 (4), pp. 685-689.

ALMANZA, C. y DACCARETT, J. (2005), Grupos estratégicos de la banca en Colombia, En: http://ciruelo.uninorte.edu.co/pdf/documentos_ieec/22/I_Documentos\%20leec\%20No \%2022.pdf.

ANSOFF, H. (1965), Corporate strategy: An analytical approach to business policy for growth and expansion. McGraw-Hill: New York.

ARISTIMUÑO, H. y ASOCIADOS (2005), La banca pequeña y mediana no cede espacio ante los líderes del sistema, En: http://www.bancaynegocios.com/noticia_ det.asp?id=2846.

BLASER, M. (2004), La banca venezolana: ¿atrapada sin salida? En: http://www. veneconomy.com/site/files/articulos/artEsp3539_2438.pdf.

BORGUCCI, E. (200I), «La concurrencia bancaria en Venezuela después de la crisis financiera de |994». Problemas del Desarrollo, 125 (32), pp. 39-70.

BRACHO, Y.; ARIZA, B.; GONZÁLEZ, M.; JIMÉNEZ, E. (2002), «Fusiones bancarias en el sistema financiero venezolano». Revista de Ciencias Sociales, 8(2), pp. 30031 I.

Bravo, E. (20 I 0), Crisis financiera, empleo y alternativas en América Latina. En: Observatorio de la Economía Latinoamericana, N. ${ }^{\circ} 129$.
CÁCERES, J. (2007), Conceptos básicos de estadística para ciencias sociales (Primera edición). Delta Publicaciones Universitarias: Madrid.

CARRASCO, S. (2009), Caso práctico ACP. En: http:// www.uv.es/ carrascs/PDF/caso ACPs.pdf

CEPAL-UNICEF (2006), Condiciones de vida: la pobreza en Venezuela. En: http://www.venescopio.org.ve/docs/ Cap7.pdf.

CHILD, J. (1997), «Strategic choice in the analysis of action, structure, organizations and environment: retrospect and prospect». Organization Studies, 18 ( I), pp. 43-76.

CLAVER, E.; MOLINA, J.; PEREIRA, J. (2006), Grupos estratégicos y su influencia en el desempeño del sector hotelero. Editorial Universitaria Ramón Areces: Madrid.

CONAPRI (Consejo Nacional de Promoción de Inversiones) (2007), Ciudades más atractivas para invertir. CONAPRI: Caracas.

COOL, K. y SCHENDEL, D. (1987). «Strategic group formation and performance. The case of the U.S. pharmaceutical industry, 1963-1982». Management Science, 33(9), pp. I | 02- I | 24.

CORREA, E.; GIRÓN, A. (2004), Economía financiera contemporánea (Volumen IV), México: UNAM.

DOMOWITZ, I.; HUBBARD, R.; PETERSEN, B. ( 1 987), «Oligopoly Supergames: Some Empirical Evidence on Prices and Margins». The Journal of Industrial Economics, 35(4),pp.379-398.

FLAVÍÁN C.; POLO,Y. ( 1999), «Hacia la homogeneización de criterios en las investigaciones de grupos estratégicos». Cuadernos de economía y dirección de la empresa, 3, pp.9-28.

FLORES, D. (2006), «Calidad y concentración de mercado en la banca comercial». Ensayos, 25 ( I),pp.21-30.

GARCÉS, L. (2007), «Grupos cognitivos (estratégicos y competitivos) en fondos de pensiones y cesantías de Colombia al 2005». Suma Psicológica, 14 ( I), pp. I07200.

GARCÉS, J. y DUQUE, E. (2008), «Grupos estratégicos en la banca colombiana: Análisis estático y dinámico». INNOVAR, Revista de Ciencias Administrativas y Sociales, I 8(32), pp. 195-226.

GÓMEZ, J.; MARHUENDA, J.; MAS, F. (1997), «El precio de las acciones como base para la identificación de grupos estratégicos: Aplicación al sector bancario español». Revista Española de Financiación y Contabilidad, 26 (92), pp. 805-828.

GUAL, J.; HERNÁNDEZ,A. ( 199 |), «Costes operativos, tamaño y especialización en las cajas de ahorro españolas». Investigaciones Económicas, I 5(3), pp.70।-726. 
GUERRA, J.y OLIVO,V. (2009), La crisis global y su impacto en Venezuela. En: http://www.fes-ecuador.org/media/ pdf/DocumentoCrisisGlobal Venezuela .pdf.

GWINNER, W.; GOLDBERG, M.; SOLO,T.; DIDONI, A. (2006), De la Exclusión a la Inclusión Financiera: Necesidad de mejorar la disponibilidad de crédito para los pobres en las zonas urbanas de América Latina. En: http://www.alide.org.pe/download/CEDOM/ Recursos_Informacion/3-INCLUSION_BANCA\%5C46Excl_ incl_enBreveNov06_98_.pdf

HITT, M.; IRELAND, R.; HOSKISSON, R. (2003), Administración estratégica: Competitividad, y conceptos de globalización (Quinta edición).Thomson International: México.

HOUTHOOFD, N. y HEENE, A. ( 1997), «Strategic groups as subsets of strategic scope groups in the Belgian brewing industry». Strategic Management Journal, 18 (8), pp. 653-666.

HUNT, M. (1972), Competition in the Mayor Home Appliance Industry, 1960-1970.Tesis Doctoral. Universidad de Harvard: USA.

KETCHEN, D.; SHOOK, C. (1996), «The application of cluster analysis in strategic management research: an analysis and critique». Strategic Management Journal, 17, pp. 44 I-458.

LEY GENERAL DE BANCOSY OTRAS INSTITUCIONES FINANCIERAS (2009), Gaceta Oficial de la República Bolivariana de Venezuela N. 5.947 (Extraordinario), fecha 23 de Diciembre de 2009.

MARTÍNEZ, D.; MILLA, A. (2005), La elaboración del plan estratégico y su implantación a través del cuadro de mando integral. Ediciones Díaz de Santos: Madrid.

MARULANDA, B. y PAREDES, M. (2006), Acceso a servicios financieros en Colombia y políticas para promoverlo a través de instituciones formales. USAID: Bogotá.

MAS, F. (1994), Competencia y dinámica de grupos estratégicos: aplicación al sector bancario español,Tesis Doctoral, España: Universidad de Alicante.

MAS, F. ( 1996), Competencia y dinámica de grupos estratégicos: Aplicación al sector bancario español, España: Universidad de Alicante.

MCNAMARA, G.; DEEPHOUSE, D.; LUCE, R. (2003), «Competitive positioning within and across a strategic group structure: the performance of core, secondary and solitary firms». Strategic Management Journal, 24, pp. $|6|-|8|$.

MEHRA, A. y FLOYD, S. (1998). «Product market heterogeneity, resource imitability and strategic group formation». Journal of Management, 24 (4), pp. 5। I-53।.

MENDIZABAL, A.; MITXEO, J.; OLASOLO, A.; ZUBIA, M. (2008), «Reflexiones sobre el origen y las implicaciones de la exclusión financiera». Universidad, Sociedad y Mercados Globales, 209-2 18.

MELANDRI, C. (2006), Banca impulsa a la microempresa. En: http://www.dinero. com.ve/2। I/portada/informe.html.

MEYER, J. y ROWAN, B. ( 1977), «Institutionalized organizations: formal structures as myth and ceremony». American Journal of Sociology, 83, pp. 340-363.

MONGAY, C. (2005), Quimiometría. Universitat de València: Valencia. España.

OCAMPO, J. (2009), «La crisis económica global: impactos e implicaciones para América Latina». Revista Nueva Sociedad, 224, pp. 48-66.

PAMPILLÓN, F.; DE LA CUESTA, M.; RUZA, C.; ARGUEDAS, R. (2004), Apuntes de tendencias del sistema financiero español (Segunda edición). Dykinson: Madrid.

PEÑA, D. (2002), Análisis de datos multivariantes. McGrawHill: Madrid.

PÉREZ, C. (2005), Métodos estadísticos avanzados con SPSS. Thomson editores: Madrid.

Porter, M. (1979), «The structure within industries and companies performance». Review of Economics and Statistics, I4, pp. 214-227.

PRIOR, D. y SURROCA, J. (200 I), Modelo para la identificación de grupos estratégicos basado en el análisis envolvente de datos: Una aplicación al sector bancario español. Barcelona: Universidad Autónoma de Barcelona.

ROCHE, J.; GIMÉNEZ, C.; FERNÁNDEZ, J.; NOGUERA, C.; TORRES, C.; DI BONAVENTURA, E.; BARROT, B.; CORREA, G. (2002), «Caracterización, Tipología y Clasificación Municipal de Venezuela: Una Medición del Desarrollo Humano a Nivel Municipal». Sistema de Información para la Gestión Local. Instituto Nacional de Estadística, Programa de las Naciones Unidas para el Desarrollo: Caracas.

ROJAS, L. (2006), El acceso a los servicios bancarios en América Latina: Identificación de obstáculos y recomendaciones. En: La Extensión del Crédito y los Servicios Financieros. Secretaría General Iberoamericana: Madrid.

RUIZ, F.; NICOLAU, J.; MAS, F. ( 1999), «Relación de los patrones de planificación y de conducta de exportación sobre los resultados»y. Dirección y organización , 22, pp. 99-1।3.

SAZA, J. (2010), Banca latinoamericana: I año después de la crisis. Federación Latinoamericana de Bancos - FELABAN. En: http://www.felaban.com/archivos_documentos_interes/DOC_CRISIS.pdf.

SOLÍS, R.; AUPING, J.; DELGADO, M.; EBRAD, M. (2000), Del Fobaproa al IPAB: Testimonios, análisis y propuestas. México: Plaza y Valdes Ed. 
SUDEBAN (2007), Boletín trimestral. Indicadores financieros: Octubre-Diciembre, 2007. Año 14, N. ${ }^{\circ} 60$.

SUDEBAN (2008), Boletín trimestral. Indicadores financieros: Abril-Junio, 2008. Año I5, N. ${ }^{\circ} 62$.

SUDEBAN (20 I I), Instituciones Bancarias Intervenidas y en Liquidación. En: http:// sudeban.gob.ve/webgui/inicio/instituciones-bancarias-intervenidas-y-en-liquidacin.

SURROCA, J. (2003), Gobierno de la empresa y eficiencia en organizaciones orientadas a los interesados: Una aplicación a las cajas de ahorro y a las cooperativas de Mondragón, Tesis Doctoral. Barcelona: Universidad Autónoma de Barcelona.

TEJERO, S. (2007), 55,7\% del activo de la banca estatal está compuesto por bonos. El Universal: Economía. En: http://noticias.eluniversal.com/2007/ 09/19/eco_art_ 55,7-del-activo-de_484378.shtml.

VERA, L. (20 I 0), ¿Por que la economía venezolana ha salido tan afectada por la crisis económica global?. Universidad Central de Venezuela. Comisión de Investigación. En: http//www.faces.ucv.ve/economia/documentos_ de_trabajo.html.

ZAHLER, R. (2008), Bancarización privada en Chile. Serie Financiamiento del Desarrollo, N. ${ }^{\circ}$ 200. CEPAL: Santiago de Chile.

ZÚÑIGA, J., DE LA FUENTE, J.; RODRÍGUEZ, J. (2004), «A study of industry evolution in the face of major environmental disturbances: group and firm strategic behaviour of Spanish banks, 1983-1997». British Journal of Management, 15 (3), pp. $219-24$. 
Anexo I

Estados y municipios en los que se encuentra al menos una oficina del GE

\begin{tabular}{|c|c|c|c|c|c|}
\hline & \\
\hline & GEl & GE2 & GE3 & GE4 & GE5 \\
\hline Total oficinas & 1.716 & 33 & 1.509 & 360 & I \\
\hline Estados en los que se encuentra al menos una oficina $\left(\mathrm{N} .^{\circ}\right)$ & $24 *$ & 9* & $24 *$ & $24 *$ & I \\
\hline Municipios en los que se encuentra al menos una oficina $\left(\mathrm{N} .^{\circ}\right)$ & 200 & 14 & 176 & 191 & I \\
\hline Cobertura geográfica** (\%) & 59,70 & 4,18 & 52,54 & 57,01 & 0,30 \\
\hline $\begin{array}{l}\text { Habitantes de la población más pequeña de un municipio } \\
\text { con oficina }\left(N .^{\circ}\right)\end{array}$ & 8.668 & 42.662 & 9.773 & 2.169 & 71.393 \\
\hline $\begin{array}{l}\text { Habitantes de la población más grande de un municipio } \\
\text { con oficina }\left(\mathrm{N} .^{\circ}\right)\end{array}$ & $\begin{array}{r}2.091 .45 \\
2\end{array}$ & $\begin{array}{r}2.091 .45 \\
2\end{array}$ & $\begin{array}{r}2.091 .45 \\
2\end{array}$ & $\begin{array}{r}2.091 .45 \\
2\end{array}$ & 71.393 \\
\hline Población promedio de los municipios con oficinas & 120.834 & 552.592 & 136.364 & 121.110 & 71.393 \\
\hline $\begin{array}{l}\text { Menor densidad poblacional de un municipio con oficina } \\
(\mathrm{Hab} . / \mathrm{Km} 2)\end{array}$ & 0,9 & 224,2 & 0,9 & 0,6 & 5.491 \\
\hline $\begin{array}{l}\text { Mayor densidad poblacional de un municipio con oficina } \\
\left(\mathrm{Hab} . / \mathrm{Km}^{2}\right)\end{array}$ & $5.816,1$ & $5.49 \mid, 8$ & $5.491,8$ & $5.491,8$ & 5.491 \\
\hline $\begin{array}{l}\text { Densidad poblacional promedio de los municipios con oficinas } \\
\left(\mathrm{Hab} / \mathrm{Km}^{2}\right)\end{array}$ & 415,5 & $2.08 \mid, 7$ & 413,9 & 362,9 & 5.491 \\
\hline
\end{tabular}

*Incluye Dtto. Federal ** de los 335 municipios del país.

Fuente: Elaboración propia a partir de datos extraídos de SUDEBAN (2008) y del INE (2007).

Anexo 2

Número de oficinas de los GE en los diez municipios menos pobres de Venezuela

\begin{tabular}{|c|c|c|c|c|c|c|c|c|c|c|c|c|c|}
\hline \multirow{2}{*}{$N .^{\circ}$} & \multirow{2}{*}{ Estado } & \multirow{2}{*}{ Municipio } & \multicolumn{2}{|c|}{ Oficinas GEI } & \multicolumn{2}{|c|}{ Oficinas GE2 } & \multicolumn{2}{|c|}{ Oficinas GE3 } & \multicolumn{2}{|c|}{ Oficinas GE4 } & \multicolumn{2}{|c|}{ Oficinas GE5 } & \multirow{2}{*}{ Total } \\
\hline & & & $N .^{\circ}$ & $\%$ & $N .^{\circ}$ & $\%$ & $N .^{\circ}$ & $\%$ & $N .^{\circ}$ & $\%$ & $N .^{\circ}$ & $\%$ & \\
\hline । & Miranda & Los Salías & 14 & 60,87 & 0 & 0,00 & 7 & 30,43 & 2 & 8,70 & 0 & 0,00 & 23 \\
\hline 2 & Anzoátegui & Diego B. Urbaneja & 16 & 53,33 & 0 & 0,00 & 13 & 43,33 & I & 3,33 & 0 & 0,00 & 30 \\
\hline 3 & Carabobo & San Diego & 10 & 52,63 & 0 & 0,00 & 9 & 47,37 & 0 & 0,00 & 0 & 0,00 & 19 \\
\hline 4 & Miranda & Chacao & 103 & 52,55 & 7 & 3,57 & 73 & 37,24 & 10 & 5,10 & 3 & 1,53 & 196 \\
\hline 5 & Miranda & Baruta & 79 & 50,32 & 0 & 0,00 & 73 & 46,50 & 5 & 3,18 & 0 & 0,00 & 157 \\
\hline 6 & Aragua & Mario B. Iragorry & । & 33,33 & 0 & 0,00 & 2 & 66,67 & 0 & 0,00 & 0 & 0,00 & 3 \\
\hline 7 & Táchira & San Cristóbal & 44 & 50,00 & I & 1,14 & 31 & 35,23 & 12 & 13,64 & 0 & 0,00 & 88 \\
\hline 8 & Lara & Palavecino & 8 & 50,00 & 0 & 0,00 & 7 & 43,75 & I & 6,25 & 0 & 0,00 & 16 \\
\hline 9 & Nva Esparta & Maneiro & 13 & 59,09 & I & 4,55 & 8 & 36,36 & 0 & 0,00 & 0 & 0,00 & 22 \\
\hline \multirow[t]{2}{*}{10} & Mérida & Libertador & 19 & 41,30 & 0 & 0,00 & 23 & 50,00 & 4 & 8,70 & 0 & 0,00 & 46 \\
\hline & & & 307 & & 9 & & 246 & & 35 & & 3 & & 600 \\
\hline
\end{tabular}

Fuente: Elaboración propia a partir de datos extraídos de SUDEBAN (2008) y CEPAL-UNICEF (2006). 
Anexo 3

Número de oficinas de los GE en los diez municipios de Venezuela más atractivos para invertir

\begin{tabular}{|c|c|c|c|c|c|c|c|c|c|c|c|c|c|}
\hline \multirow{2}{*}{$N .^{\circ}$} & \multirow{2}{*}{ Estado } & \multirow{2}{*}{ Municipio } & \multicolumn{2}{|c|}{ Oficinas GEI } & \multicolumn{2}{|c|}{ Oficinas GE2 } & \multicolumn{2}{|c|}{ Oficinas GE3 } & \multicolumn{2}{|c|}{ Oficinas GE4 } & \multicolumn{2}{|c|}{ Oficinas GE5 } & \multirow{2}{*}{ Total } \\
\hline & & & $N .^{\circ}$ & $\%$ & $N .^{\circ}$ & $\%$ & $N .^{\circ}$ & $\%$ & $N^{\circ}$ & $\%$ & $N^{\circ}$ & $\%$ & \\
\hline I & Dtto. Federal & Libertador & 210 & 43,66 & 5 & 1,04 & 223 & 46,36 & 43 & 8,94 & 0 & 0 & 481 \\
\hline 2 & Carabobo & Valencia & 66 & 42,86 & 4 & 2,60 & 76 & 49,35 & 8 & 5,19 & 0 & 0 & 154 \\
\hline 3 & Táchira & San Cristóbal & 44 & 50,00 & । & 1,14 & 31 & 35,23 & 12 & 13,64 & 0 & 0 & 88 \\
\hline 4 & Aragua & Girardot & 59 & 56,73 & 3 & 2,88 & 36 & 34,62 & 6 & 5,77 & 0 & 0 & 104 \\
\hline 5 & Zulia & Maracaibo & 62 & 31,16 & 3 & $|, 5|$ & 124 & 62,31 & 10 & 5,03 & 0 & 0 & 199 \\
\hline 6 & Mérida & Libertador & 19 & 41,30 & 0 & 0,00 & 23 & 50,00 & 4 & 8,70 & 0 & 0 & 46 \\
\hline 7 & Nva Esparta & Mariño & 22 & 59,46 & 2 & 5,41 & 11 & 29,73 & 2 & 5,41 & 0 & 0 & 37 \\
\hline 8 & Bolívar & Caroní & 76 & 62,81 & 0 & 0,00 & 36 & 29,75 & 9 & 7,44 & 0 & 0 & 121 \\
\hline 9 & Falcón & Carirubana & 20 & 50,00 & 0 & 0,00 & 17 & 42,50 & 3 & 7,50 & 0 & 0 & 40 \\
\hline \multirow[t]{2}{*}{10} & Lara & Iribarren & 83 & 57,64 & I & 0,69 & 52 & 36,11 & 8 & 5,56 & 0 & 0 & 144 \\
\hline & & & 661 & & 19 & & 629 & & 105 & & 0 & 0 & 1414 \\
\hline
\end{tabular}

Fuente: Elaboración propia a partir de datos extraídos de SUDEBAN (2008) y CONAPRI (2007).

Anexo 4

Número de oficinas de los GE en los cinco municipios menos poblados de Venezuela

\begin{tabular}{|c|c|c|c|c|c|c|c|c|c|c|c|c|c|}
\hline \multirow{2}{*}{$N .^{\circ}$} & \multirow{2}{*}{ Estado } & \multirow{2}{*}{ Municipio } & \multicolumn{2}{|c|}{ Oficinas GEI } & \multicolumn{2}{|c|}{ Oficinas GE2 } & \multicolumn{2}{|c|}{ Oficinas GE3 } & \multicolumn{2}{|c|}{ Oficinas GE4 } & \multicolumn{2}{|c|}{ Oficinas GE5 } & \multirow{2}{*}{ Total } \\
\hline & & & $\mathrm{N}^{\circ}$ & $\%$ & $\mathrm{~N}^{\circ}{ }^{\circ}$ & $\%$ & $N .^{\circ}$ & $\%$ & $N^{\circ}$ & $\%$ & $\mathrm{~N}^{\circ}{ }^{\circ}$ & $\%$ & \\
\hline I & Amazonas & Rio Negro & 0 & 0,00 & 0 & 0,00 & 0 & 0,00 & 0 & 0,00 & 0 & 0,00 & 0 \\
\hline 2 & Mérida & Padre Noguera & 0 & 0,00 & 0 & 0,00 & 0 & 0,00 & । & 100,00 & 0 & 0,00 & । \\
\hline 3 & Trujillo & José F. Márquez & 0 & 0,00 & 0 & 0,00 & 0 & 0,00 & 0 & 0,00 & 0 & 0,00 & 0 \\
\hline 4 & Táchira & Simón Rodríguez & 0 & 0,00 & 0 & 0,00 & 0 & 0,00 & I & 100,00 & 0 & 0,00 & I \\
\hline 5 & Falcón & Palma Sola & 0 & 0,00 & 0 & 0,00 & 0 & 0,00 & 0 & 0,00 & 0 & 0,00 & 0 \\
\hline \multicolumn{3}{|c|}{ Total $\mathrm{N}^{\circ}$ de oficinas del GE } & 0 & & 0 & & 0 & & 2 & & 0 & & 2 \\
\hline
\end{tabular}

Fuente: Elaboración propia a partir de datos extraídos de SUDEBAN (2008) y de Roche et al. (2002). 
Anexo 5

Número de oficinas de los GE en los diez municipios más pobres de Venezuela

\begin{tabular}{|c|c|c|c|c|c|c|c|c|c|c|c|c|c|}
\hline \multirow{2}{*}{$N .^{\circ}$} & \multirow{2}{*}{ Estado } & \multirow{2}{*}{ Municipio } & \multicolumn{2}{|c|}{ Oficinas GE I } & \multicolumn{2}{|c|}{ Oficinas GE2 } & \multicolumn{2}{|c|}{ Oficinas GE3 } & \multicolumn{2}{|c|}{ Oficinas GE4 } & \multicolumn{2}{|c|}{ Oficinas GE5 } & \multirow{2}{*}{ Total } \\
\hline & & & $N .^{\circ}$ & $\%$ & $\mathrm{~N}^{\circ}$ & $\%$ & $N .^{\circ}$ & $\%$ & $N .^{\circ}$ & $\%$ & $\mathrm{~N}^{\circ}$ & $\%$ & \\
\hline । & Delta Amacuro & Antonio Díaz & 0 & 0,00 & 0 & 0,00 & 0 & 0,00 & 0 & 0,00 & 0 & 0,00 & 0 \\
\hline 2 & Trujillo & Monte Carmelo & 0 & 0,00 & 0 & 0,00 & 0 & 0,00 & 0 & 0,00 & 0 & 0,00 & 0 \\
\hline 3 & Mérida & Justo Briceño & 0 & 0,00 & 0 & 0,00 & 0 & 0,00 & 0 & 0,00 & 0 & 0,00 & 0 \\
\hline 4 & Barinas & Arismendi & 0 & 0,00 & 0 & 0,00 & 0 & 0,00 & । & 100,00 & 0 & 0,00 & । \\
\hline 5 & Guárico & San G. de Guayabal & 0 & 0,00 & 0 & 0,00 & 0 & 0,00 & । & 100,00 & 0 & 0,00 & । \\
\hline 6 & Mérida & Cardenal Quintero & 0 & 0,00 & 0 & 0,00 & 0 & 50,00 & । & 50,00 & 0 & 0,00 & 2 \\
\hline 7 & Delta Amacuro & Pedernales & 0 & 0,00 & 0 & 0,00 & 0 & 0,00 & 0 & 0,00 & 0 & 0,00 & 08 \\
\hline 8 & Barinas & Andrés Eloy Blanco & 0 & 0,00 & 0 & 0,00 & 0 & 0,00 & । & 100,00 & 0 & 0,00 & । \\
\hline 9 & Barinas & Rojas & 0 & 0,00 & 0 & 0,00 & । & 33,33 & 2 & 66,67 & 0 & 0,00 & 3 \\
\hline 10 & Barinas & Sosa & 0 & 0,00 & 0 & 0,00 & 0 & 0,00 & I & 100,00 & 0 & 0,00 & I \\
\hline \multicolumn{3}{|c|}{ Total $N^{\circ}$ de oficinas del GE } & 0 & & 0 & & 2 & & 7 & & 0 & & 9 \\
\hline
\end{tabular}

Fuente: Elaboración propia a partir de datos extraídos de SUDEBAN (2008) y CEPAL-UNICEF (2006). 